\title{
Subacute brain-stem encephalitis
}

\author{
S. G. WAXMAN ${ }^{1}$, T. D. SABIN, AND L. J. EMBREE \\ From the Neurological Unit and the Mallory Institute of Pathology, Boston City Hospital, \\ and Departments of Neurology, Harvard Medical School and Tufts Medical School, Boston, \\ Massachusetts, U.S.A.
}

SYNOPSIS A 65 year old man developed progressive signs of pontine and medullary dysfunction with striking bilateral paralysis of lateral gaze, dysarthria, dysphagia, and ataxia. A respiratory death occurred seven months from the onset. Pathological examination revealed focal brain-stem changes of perivascular lymphocytic cuffing, microglial infiltration, glial nodules, and neuronophagia. No underlying malignancy or general disease impairing immunity mechanisms was discovered.

This report concerns a case of subacute brainstem encephalitis in a 65 year old male. The patient presented a distinct clinical picture of progressive dysfunction of structures on the floor of the IVth ventricle with marked involvement of lateral gaze centres. Clinical localization was precisely reflected by the distribution of pathological changes, suggesting a pathological entity which might be liable to antemortem diagnosis. The failure to discover a malignancy or immunological disorder is another singular feature of this case of subacute focal encephalitis.

\section{CASE REPORT}

(BCH no. 2239114) This 65 year old right-handed white male was admitted on 18 January 1973 for evaluation of progressive bulbar dysfunction. He smoked two packs of cigarettes a day and had a history of mild chronic emphysema, but was otherwise in good general health until July 1972, when he sustained a blow to the left frontal region while entering a car. There was no laceration or unconsciousness, but he complained of generalized headache for 24 hours. Ten days later he noted double vision, intermittent nausea, and unsteadiness of gait. He conceded that these symptoms might have appeared transiently in the week before his minor head injury. On 30 July 1972 he was admitted to another hospital and noted to have a blood pressure of $140 / 95 \mathrm{mmHg}$, vertical nystagmus on

1 Address for correspondence: Dr Stephen Waxman, Neurological Unit (Medical 9), Boston City Hospital, 818 Harrison Avenue, Boston, Massachusetts 02118, U.S.A. upward gaze, and slight rotatory nystagmus on left lateral gaze. There was mild unsteadiness on tandem gait. Red glass testing suggested decreased motility of the left eye. Routine blood and urine studies were normal. Radiographs of the chest showed a tortuous aorta, while skull films were normal with a midline pineal shadow. Left carotid and vertebral angiograms were normal. Lumbar puncture yielded 'clear fluid with normal pressure, glucose, and protein, and contained 7 lymphocytes per $\mathrm{cm}$ '. Thyroid scan showed a solitary cold nodule and a T3 and T4 uptake were normal. In September 1972 the patient noted difficulty in chewing, swallowing food, and managing secretions. His speech had become slurred. Examination showed a complete paralysis of right lateral gaze, dysarthria, and ataxia. An oesophogram revealed neuromuscular dysfunction in swallowing with ineffectual clearing of valleculae and pyriform sinuses and diminished peristaltic activity of the upper oesophagus. Electroencephalography, brain scan, intravenous pyelography, barium enema, and radiographic bone series for metastases were normal. Spinal fluid protein was $21 \mathrm{mg}$ per $100 \mathrm{ml}$ with negative cytology. A pneumoencephalogram was normal and demonstrated that the fourth ventricle was not displaced and the floor had a normal contour.

The symptoms of brain-stem dysfunction progressed and by December 1972 nasal regurgitation of fluids and stiffness in opening of the mouth were noted. New findings included right lid retraction and facial weakness, incomplete left eye abduction, tongue weakness, and decreased hearing on the right. The jaw jerk was brisk. A week of high dosage steroids had no effect.

On 18 January 1973 the patient was admitted to 
the Neurological Unit. Vital signs were normal; aegophony and decreased breath sounds were detected at the base of the right chest. Mental status was normal. Visual acuity corrected to $20 / 40$ bilaterally and a concentric constriction of the right visual field was suspected. The fundi were normal. The patient was unable to make any conjugate lateral eye movement to either side on command. Reinforcement by the oculocephalic manoeuvre with maintained visual fixation effected only a few degrees of O.D. adduction and no movements of O.S. Vertical excursions were adequate. On upward gaze, vertical jerk nystagmus appeared, and downward gaze was frequently interrupted by upward bobbing movements, which also appeared during efforts at lateral gaze. Lateral optokinetic nystagmus was absent. The pupils were normal. The sensory trigeminal functions were intact but marked weakness in jaw opening and closure was present. There was mild weakness in upper and lower portions of the right face. Taste perception was normal. Moderate impairment of high tone hearing was noted on the right. The Weber test did not lateralize and air conduction was greater than bone conduction of sound bilaterally. While there were poor palatal movements on phonation, there was a very active gag reflex without deviation of the median raphe of the palate. The patient was not hoarse. Sternomastoid and trapezius strength were normal. The tongue was weak and protruded incompletely but without deviation. Occasional fasciculations appeared at the base of the tongue on the left but no atrophy was seen. Normal strength and tone were present in the extremities. There was mild side-to-side proximal tremor in the right arm on finger-nose testing. Cerebellar tremor was more prominent on the right in heel-shin testing. The gait was ataxic with a tendency to fall to the left. The reflexes were normally active and equal but the left plantar response was extensor. Sensation was normal. Haemogram, urinalysis, serum electrolytes, blood sugar, and liver function tests were normal. Radiograph of the chest showed a lingular infiltrate and prominent left hilum. Lumbar puncture (opening pressure $130 \mathrm{~mm}$ ) yielded clear, colourless fluid with protein $36 \mathrm{mg}$ and sugar $126 \mathrm{mg}$ per $100 \mathrm{ml}$, no leucocytes, 412 erythrocytes per $\mathrm{mm}^{3}$ in the first tube and 38 per $\mathrm{mm}^{3}$ in the third tube. On the second hospital day the patient complained that he felt as though he was 'running out of breath'. Respiratory rate was 16 per min and arterial blood gases had a $\mathrm{pCO}_{2}$ of $55 \mathrm{mmHg}, \mathrm{pO}_{2} 68 \mathrm{mmHg}$, and $\mathrm{pH} 7 \cdot 43$. On the following day he vomited coffee ground material and suffered a respiratory arrest. He remained comatose with decortication and no spontaneous respiratory activity until his death on the fourth hospital day.

GENERAL PATHOLOGICAL FINDINGS On postmortem examination there were fractured ribs due to resuscio tation, three small superficial mucosal haemorrhages in the stomach, and pulmonary congestion and oedema (lungs: $1100 \mathrm{~g}$ ) with pulmonary vasculat sclerosis. There were subpleural blebs measuring from $0.4 \mathrm{~cm}$ to $4.0 \mathrm{~cm}$ on the lungs, with pleural

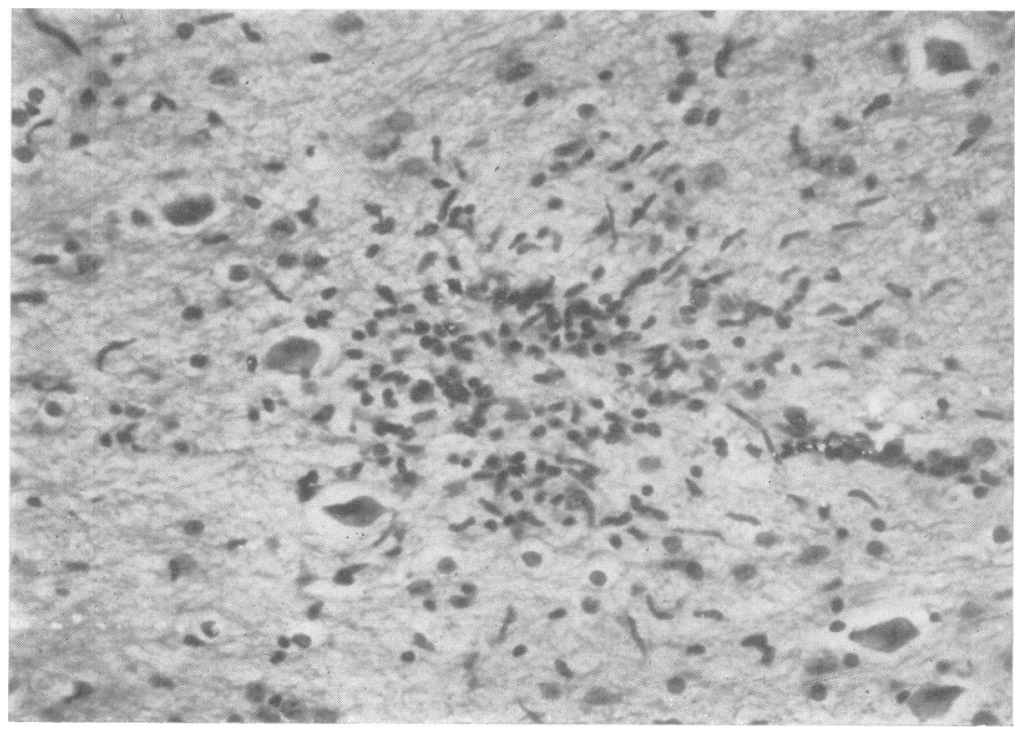

FIG. 1. Microglial nodule from the left inferior olive. The inferior olives were the site of many glial nodules; microgliosis and neuronophagia were also present. Haematoxylin and eosin, $\times 200$. 


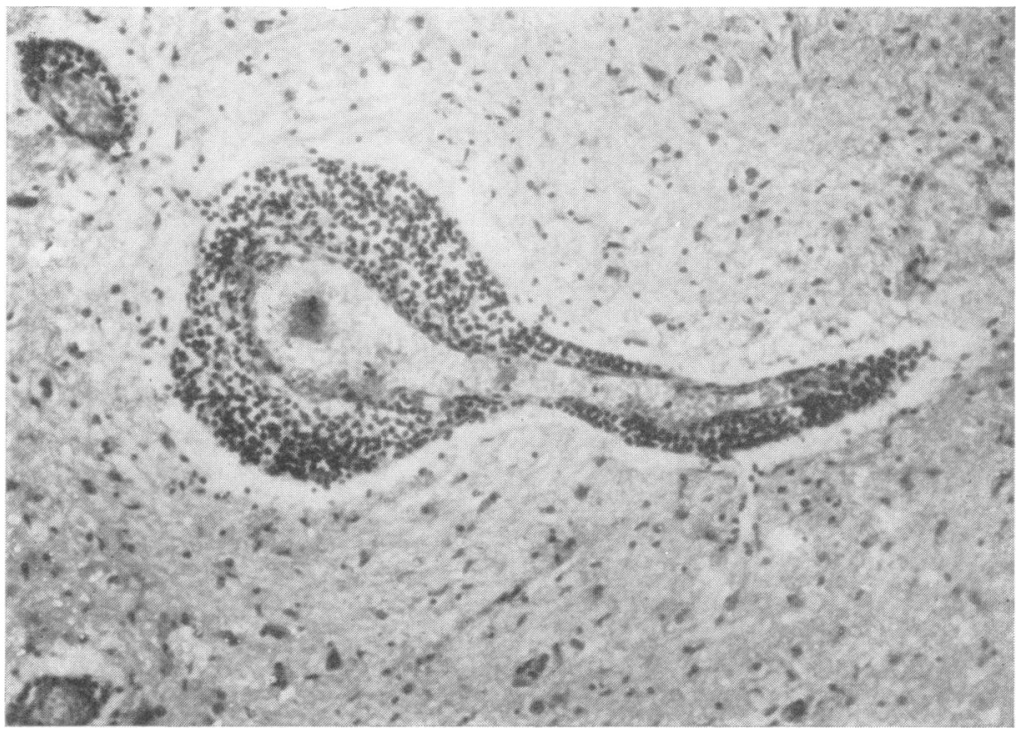

FIG. 2. Perivascular cuffing within the hypoglossal nucleus. Haematoxylin and eosin, $\times 100$.

adhesions between the left lower lobe and chest wall. Careful examination of the lungs did not reveal carcinoma, and no other systemic neoplasm was demonstrable.

NEUROPATHOLOGICAL OBSERVATION On gross examination, the calvarium, base of the skull, and meninges were normal. The brain weighed $1340 \mathrm{~g}$. There was minimal arteriosclerotic narrowing of the carotid arteries. The vertebrobasilar system was normal. There was bilateral herniation of the cerebellar tonsils, with encephalomalacia of the left calcarine cortex and superior lateral cerebellar cortex. The brain-stem appeared normal externally. No

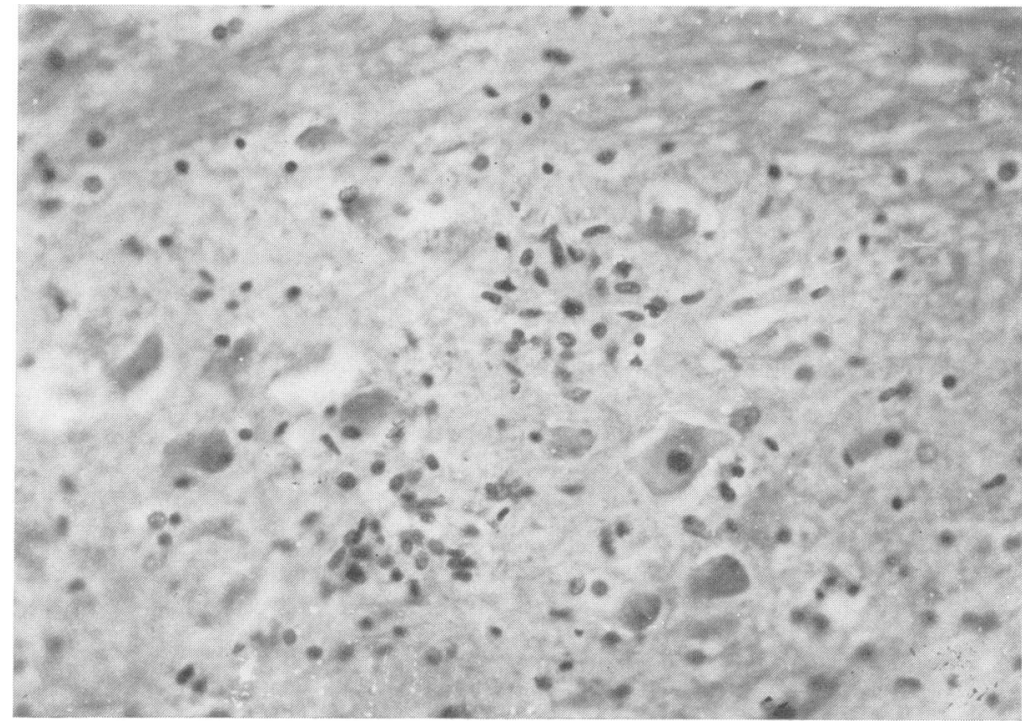

FIG. 3. Pontine tegmentum.

The neurones are deeply stained and angular, and there is evidence of neuronophagia. Haematoxylin and eosin, $\times 200$. 


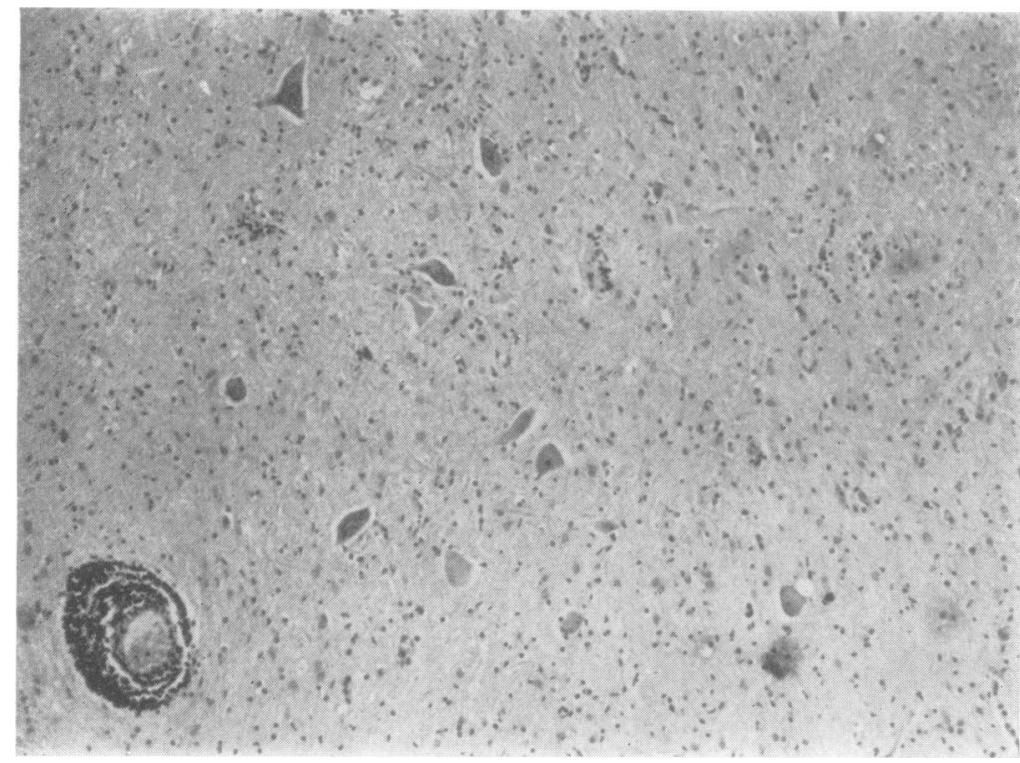

FIG. 4. Facial nucleus

(right). The remaining neurones are deeply eosinophilic and display karyorrhexus, and a glial nodule is present. Note also the perivascular cuffing. Haematoxylin and eosin, $\times 60$.

abnormalities were evident on inspection of cut surfaces through the midbrain, pons, or medulla. Ventricles were normal size.

Histological examination revealed marked, largely lymphocytic, perivascular cuffing, with intense microglial infiltration observed from the upper spinal cord to midbrain but most obvious in the pontine tegmentum. Loss of neurones, neuronophagia, and glial nodules were present. Involvement was not confined to any single vascular, morphogenetic, or functional distribution. The spinal cord at $\mathrm{Cl}$ segmental level exhibited mild microgliosis, perivascular cuffing, and loss of motoneurones on the right side, with a normal histological picture on the left. Spinal nucleus of $\mathrm{V}$ appeared normal on both sides. The cells of the inferior olives were shrunken and eosinophilic; there were perivascular cuffing, neuronophagia, and microgliosis, with numerous glial nodules (Fig. 1). Gracile and cuneate nuclei were relatively spared. Hypoglossal nuclei showed neuronal loss, glial nodules, and cuffing of vessels (Fig. 2). The medial vestibular nuclei bilaterally, right lateral vestibular nucleus, and dorsal nuclei of the vagi were sites of neuronal loss. Nucleus ambiguus appeared normal on the left but could not be identified on the right. Examination of the pontine tegmentum showed neuronophagia and marked microgliosis in the pontine nuclei (Fig. 3). Facial nuclei were depopulated bilaterally (Fig. 4), as were the abducens nuclei. Trochlear nuclei were normal. Signs of inflammation tapered off at the ponto- mesencephalic level; the mesencephalic tectum an tegmentum showed only traces of perivascular cuffini and microgliosis. Oculomotor nuclei exhibited some microgliosis but the neurones appeared normat Substantia nigra and red nucleus were unremarkable Sections stained for myelin revealed no loss of myelin anywhere in the brain-stem, even in areas of striking neuronophagia and microgliosis. Corticospinal and pontocerebellar fibres appeared normal More rostral areas exhibited a picture consistent with severe ischaemic changes, but no perivascular cuffing, microglial infiltration, or neuronophagia. Cerebellar Purkinje cells were deeply eosinophilic and had angular outlines with occasional karyorrhexis. The cerebellar white matter showed scattered areas of thinning of myelin with reactive astrocytosis. Diencephalic structures exhibited loss of neurones and eosinophilia, with angularity of remaining neurones in the putamen and globus pallidus. In the hippocampus there was a paucity of pyramidal cells; those remaining were deeply eosinophilic. Capillary endothelial cells were swollen. There was pseudolaminar necrosis of the parahippocampal cortex. No senile plaques or neurofibrillary tangles were observed in Bodian preparations. Motor and sensory cerebral cortex exhibited pseudolaminar necrosis Anoxic changes were also present in orbitofrontal and calcarine cortex, with severe pseudolaminar necrosis in the left calcarine cortex. There was diffuse chronic leptomeningeal thickening, with macro- 
phages, monocytes, and rare polymorphonuclear granulocytes in the subarachnoid space.

\section{DISCUSSION}

While the nature of this patient's disease eluded diagnosis throughout the seven month course and at the macroscopic examination of the brain, microscopic examination revealed an encephalitis severely affecting, but not extending more rostral than, the brain-stem. More rostral changes, most marked in the calcarine cortex and the cerebellum, were characteristic of anoxic damage. These findings, taken together with the patient's seven months course of gradually progressing neurological involvement, indicate that the pathology was that of a subacute brain-stem encephalitis.

Henson et al. (1965) reported a series of 10 cases of encephalomyelitis with carcinoma. In eight cases there was pathological evidence of brain-stem involvement, and in five there were only minimal changes rostral to the brain-stem. As in the present patient, inflammatory changes were most intense in the medulla and floor of the fourth ventricle. Duration of neurological symptoms in these five patients ranged from six to 20 months. In contrast with the present case, three of their patients presented with muscle weakness in lower extremities and on pathological examination demonstrated involvement of the spinal cord with loss of anterior horn cells. The fourth patient presented with paraesthesia of the right hand, with lymphocytic infiltration of posterior root ganglia and involvement of posterior columns found at postmortem examination. The fifth patient complained of pulmonary symptoms for four years and generalized muscle weakness for six months, with involvement at postmortem examination limited to the subthalamic area, substantia nigra, and inferior olives. Each of the patients had carcinoma (one involving the breast, four bronchial) and in four cases the diagnosis of carcinoma was made clinically. A case of encephalomyelitis involving cervical spinal cord and brainstem in a patient with occult oat-cell carcinoma of the lung was discussed by Adams (1970). In this case, the history of dysphagia began three years before death. There was weakness, loss of hearing, and loss of temperature and pain sensa- tion between the second and fifth cervical segments, but there was no abnormality of extraocular movements. Subacute focal adenovirus encephalitis involving the occipital lobe in a patient on immunosuppressive therapy has also been described (Chou et al., 1973). Varicellazoster encephalomyelitis has been reported in a patient with leukaemia treated with immunosuppressant drugs and radiation (McCormick et al., 1969). Walton et al. (1968) reported a case with chronic inflammation of anterior horn cells, possibly due to viral infection, producing progressive muscular weakness and wasting in a patient with Hodgkin's disease treated with radiotherapy and immunosuppressive agents.

Subacute focal encephalitides need not necessarily occur in the setting of carcinoma or immunological disorder. Brierley et al. (1960) described three cases of subacute encephalitis in later adult life. Limbic areas were most severely involved, but in one case the inferior olives showed widespread loss of nerve cells with microglial and astrocytic proliferation and infiltration of vessel walls. In two of these cases no carcinoma was present; in the third, several mediastinal lymph nodes were found at necropsy to be replaced by tissue similar to that seen in bronchial carcinoma, but no primary growth was found.

The present case emphasizes the subacute course and localized involvement in some encephalitides. The distinct clinical picture, together with the pathological observations, suggest that in future cases the diagnosis of subacute brain-stem encephalitis might be made on clinical grounds. Absence of signs suggesting neoplastic disease or immunological disorder appears not to be inconsistent with the diagnosis. Virological studies of future cases may well be revealing.

The authors wish to thank Dr John T. Hornblow for allowing us to evaluate his patient and Professor Norman Geschwind for his helpful comments and discussions. This work was supported in part by grants from the National Institutes of Health.

\section{REFERENCES}

Adams, R. D. (1970). Case records of the Massachusetts General Hospital Case 42-1970. New England Journal of Medicine, 283, 806-814. 
Brierley, J. B., Corsellis, J. A. N., Hierons, R., and Nevin, S. (1960). Subacute encephalitis of later adult life mainly affecting the limbic areas. Brain, 83, 357-368.

Chou, S. M., Roos, R., Burrell, R., Gutmann, L., and Harley, J. B. (1973). Subacute focal adenovirus encephalitis. Journal of Neuropathology and Experimental Neurology, 32, 34-50.
Henson, R. A., Hoffman, H. L., and Urich, H. (1965). \ Encephalomyelitis with carcinoma. Brain, 88, 449-464.

McCormick, W. F., Rodnitzky, R. L., Schochet, S. S., Jr, and McKee, A. P. (1969). Varicella-zoster encephalomyelitis. Archives of Neurology (Chic.), 21, 559-570.

Walton, J. N., Tomlinson, B. E., and Pearce, G. W. (1968). Subacute 'poliomyelitis' and Hodgkin's disease. Journal of the Neurological Sciences, 6, 435-445. 